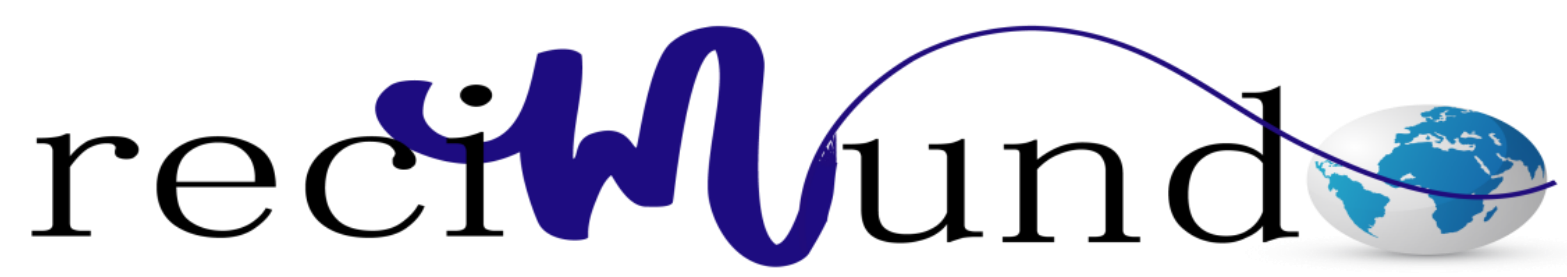

Revista Cientifica Mundo de la Investigación y el Conocimiento

Denisse Maricela Salcedo Aparicio a; Auria Lorenza Salcedo Aparicio ${ }^{\text {b }}$; Joseline Elizabeth Pazmiño Peñafiel ${ }^{c}$

Cuadro comparativo de estudiantes con súper dotación en las carreras de humanidades en las universidades de Perú y Ecuador

Comparative chart of students with super endowment in the humanities careers in the universities of Peru and Ecuador

Revista Científica Mundo de la Investigación y el Conocimiento. Vol. 3 núm.3. Esp., noviembre, ISSN: 2588-073X, 2019, pp. 817-833

DOI: 10.26820/recimundo/3.(3.Esp).noviembre.2019.817-833

URL: http://recimundo.com/index.php/es/article/view/631

Código UNESCO: 5802 Organización y Planificación de la Educación

Tipo de Investigación: Artículo de Revisión

(C) RECIMUNDO; Editorial Saberes del Conocimiento, 2019

Recibido: $15 / 09 / 2019$

Aceptado: 23/10/2019

Publicado: 30/11/2019

Correspondencia: denisse.salcedoa@ug.edu.ec

a. Magister en Educación Informática; Licenciado en Ciencias de la Educación con Especialización en Informática; Psicóloga Clínica; Universidad de Guayaquil; Guayaquil, Ecuador; denisse.salcedoa@ug.edu.ec

b. Magister en Diseño Curricular; Licenciada en Ciencias de la Educación Especialización Educación Primaria; Profesora de Segunda Enseñanza Especialización Lengua Inglesa y Lingüística; Profesor de Educación Primaria Nivel Tecnológico; Ministerio de Educación; Guayaquil, Ecuador; aurimadly@yahoo.com

c. Master Universitario en Psicopedagogía; Licenciada en Ciencias de la Educación mención Educación Básica; Ministerio de Educación; Guayaquil, Ecuador; joselyn_eli@hotmail.com 


\section{Cuadro comparativo de estudiantes con súper dotación en las carreras de humanidades en las universidades de Perú y Ecuador}

Vol. 3, núm. 3 Esp., (2019)

Denisse Maricela Salcedo Aparicio; Auria Lorenza Salcedo Aparicio; Joseline Elizabeth Pazmiño Peñafiel

\section{RESUMEN}

Un superdotado es aquella persona cuyas capacidades son superiores a las normales, o a las esperadas para su edad y condición, en una o en varias áreas de la conducta humana. Uno de los objetivos más importantes de los programas para los superdotados y talentosos es ayudarle a descubrir sus capacidades, que descubran los ámbitos más apropiados para su futuro profesional y científico. Los test de inteligencia pueden predecir el éxito escolar, pero no el éxito profesional o el éxito en la vida, pues para esto se requieren otras condiciones, como el juicio social, la empatía, la tolerancia a la frustración, la motivación, el autocontrol, la capacidad de esfuerzo y las habilidades de comunicación y de resolución de problemas. En Perú con la finalidad de propiciar espacios que faciliten la emergencia de los talentos a lo largo de las diferentes etapas educativas, y tomando en cuenta la necesidad de generar un capital humano de excelencia en aprendizaje e innovación para ser competitivos a nivel internacional, se ha propuesto el proyecto Creatividad, Innovación y Talento (CIT) que reorienta los espacios de intervención, desde el aula de clases en la escolaridad primaria y secundaria, hasta la convocatoria de tipo concurso en la educación superior. Los estudiantes de Ecuador tienen prevalencia al límbico derecho mostrando dominio en áreas afines a la comunicación, inteligencia emocional, carisma y liderazgo para mover masas, este tipo de estudiantes se perfilan para ser políticos, comunicadores sociales, educadores, investigadores. La metodología usada es descriptiva, con un enfoque documental, es decir, revisar fuentes disponibles en la red, como google académico, con contenido oportuno y relevante desde el punto de vista científico que enriquezca el análisis del tema planteado en este artículo.

Palabras claves: Superdotado; Lucha; Destrezas; Diferentes; Aprendizaje; Calidad de la educación; Docentes de vanguardia; Retos. 


\title{
Cuadro comparativo de estudiantes con súper dotación en las carreras de humanidades en las universidades de Perú y Ecuador
}

Vol. 3, núm. 3 Esp., (2019)

Denisse Maricela Salcedo Aparicio; Auria Lorenza Salcedo Aparicio; Joseline Elizabeth

Pazmiño Peñafiel

\begin{abstract}
A gifted person is a person whose abilities are superior to normal, or to those expected for their age and condition, in one or several areas of human behavior. One of the most important objectives of the gifted and talented programs is to help you discover your abilities, to discover the most appropriate areas for your professional and scientific future. The intelligence tests can predict school success, but not professional success or success in life, because this requires other conditions, such as social judgment, empathy, and tolerance for frustration, motivation, selfcontrol, the capacity for effort and communication and problem solving skills. In Peru, in order to promote spaces that facilitate the emergence of talents throughout the different educational stages, and taking into account the need to generate a human capital of excellence in learning and innovation to be competitive internationally, it has been Proposed the Creativity, Innovation and Talent (CIT) project that reorients intervention spaces, from the classroom in primary and secondary schooling, to the call for competition in higher education. Ecuadorian students have a prevalence in the limbic right showing dominance in areas related to communication, emotional intelligence, charisma and leadership to move masses, these types of students are emerging to be politicians, social communicators, educators, researchers. The methodology used is descriptive, with a documentary approach that is, reviewing sources available on the web, such as google scholar, with timely and scientifically relevant content that enriches the analysis of the topic raised in this article.
\end{abstract}

Keywords: Gifted; Struggle; Skills; Different; Learning; Quality of education; Avant-garde teachers; Challenges. 


\section{Cuadro comparativo de estudiantes con súper dotación en las carreras de humanidades en las universidades de Perú y Ecuador}

Vol. 3, núm. 3 Esp., (2019)

Denisse Maricela Salcedo Aparicio; Auria Lorenza Salcedo Aparicio; Joseline Elizabeth Pazmiño Peñafiel

\section{Introducción.}

En el proceso de enseñanza-aprendizaje universitario los profesores se enfrentan, en su día a día, a las peculiaridades de sus alumnos para interiorizar los contenidos de las disciplinas docentes, las cuales se manifiestan en la forma de estudiar, de tomar notas de clases, de participar de manera activa o pasiva en el aula, en las dificultades para aprender determinados conocimientos $y$ hasta en la facilidad para apropiárselos debido a altas potencialidades intelectuales en algunos de ellos. Ante estas situaciones las medidas que adoptan los docentes, en ocasiones, responden a criterios empíricos, con una limitada fundamentación científica debido a una insuficiente preparación teórica para enfrentar exitosamente esta complejidad psicológica en la actividad docente.

Se calcula que podrían existir más de medio millón de estos niños en Perú, teniendo como referencia a personas con un coeficiente intelectual de más de 130 puntos, cuando el promedio es de 100 puntos. Especialistas refieren que la superdotación consiste en un conjunto de factores intelectuales que posibilitan una producción general significativamente distinta de la del grupo normal; mientras que una persona talentosa tiene una capacidad centrada en un aspecto cognitivo o destreza conductual específica (Arbaccó, 2019). En Ecuador se estima que hay un 2,5\% de la población que obtiene un puntaje alto, superior a los 130 mencionados anteriormente.

Otro elemento clave en el desarrollo de estos jóvenes es la familia, juega un papel primordial en la socialización de los estudiantes superdotados por cuanto necesitan del apoyo afectivo que les permita tener seguridad en sí mismos. No siempre cuentan con el apoyo familiar 


\section{Cuadro comparativo de estudiantes con súper dotación en las carreras de humanidades en las universidades de Perú y Ecuador}

Vol. 3, núm. 3 Esp., (2019)

Denisse Maricela Salcedo Aparicio; Auria Lorenza Salcedo Aparicio; Joseline Elizabeth

Pazmiño Peñafiel

ni en las universidades cuyas clases diseñadas para un colectivo pueden acarrear desanimo en estos jóvenes, llenos de energía, sed por aprender y hambre de nuevos retos.

En este artículo se explicará brevemente concepto, características, cifras de personas cuantificadas como superdotados en fuentes de artículos científicos y académicos. Son muchos los retos por superar e investigar en esta fascinante temática.

\section{Metodología.}

Esta investigación está dirigida al estudio del "Cuadro comparativo de estudiantes con súper dotación en las carreras de humanidades en las universidades de Perú y Ecuador”. Para realizarlo se usó una metodología tipo descriptiva, con un enfoque documental, es decir, revisar fuentes disponibles en la red, como google académico, con contenido oportuno y relevante desde el punto de vista científico para dar respuesta a lo tratado en el presente artículo y que sirvan de inspiración para realizar otros proyectos. Las mismas pueden ser consultadas al final, en la bibliografía.

\section{Resultados.}

Un superdotado es aquella persona cuyas capacidades son superiores a las normales, o a las esperadas para su edad y condición, en una o en varias áreas de la conducta humana. Esta definición, aparentemente clara y sencilla, abre, desde el punto de vista educativo, una serie de interrogantes no fáciles de resolver, tales como: ¿qué es lo normal y lo anormal?, ¿cómo constatar esas diferencias?, ¿en qué medida deben existir esas deferencias para requerir tratamiento especializado? La multitud de posibles respuestas queda claramente reflejada en la 


\section{Cuadro comparativo de estudiantes con súper dotación en las carreras de humanidades en las universidades de Perú y Ecuador}

Vol. 3, núm. 3 Esp., (2019)

Denisse Maricela Salcedo Aparicio; Auria Lorenza Salcedo Aparicio; Joseline Elizabeth Pazmiño Peñafiel

falta de unanimidad científica en cuanto al concepto y a la terminología, en la multitud de enfoques y métodos utilizados en las diversas disciplinas y por los diversos especialistas (pedagogos, maestros, psicólogos, médicos etc.) en la atención a estos sujetos. Es completamente distinto partir de la idea de considerar la superdotación como manifestación de alguna capacidad por encima de la media, en cuyo caso el número de posibles "dotados" podría llegar hasta un $20 \%$ de la población (Martínez, 2019), que considerar que estos sujetos serían aquellos que mediante pruebas demostrables de capacidad se encontraran en el límite superior de la distribución de la curva normal, aquí la proporción oscilaría en cifras mucho menores, de un $2 \%$ a un $5 \%$ como máximo, o considerar únicamente a los sujetos situados en el $1 \%$ superior en inteligencia dentro de la población total. Para numerosos autores el concepto "superdotado" es un término genérico, contendría muchas manifestaciones distintas de la capacidad superior. Algunas de las acepciones del concepto se muestran a continuación:

- Precoces: los que tienen un desarrollo temprano inusual para su edad. La mayoría de los superdotados son precoces, pero no todos los niños precoces llegan a desarrollar capacidades excepcionales.

- Prodigios: son los que realizan una actividad fuera de lo común para su edad y condición. Desarrollan productos que llaman la atención en un campo específico, memoria, lenguaje, ritmo etc. Ha habido figuras eminentes que fueron niños prodigio en su infancia; el caso de Mozart es universalmente conocido, pero ha habido otros que no lo fueron Einstein o 


\section{Cuadro comparativo de estudiantes con súper dotación en las carreras de humanidades en las universidades de Perú y Ecuador}

Vol. 3, núm. 3 Esp., (2019)

Denisse Maricela Salcedo Aparicio; Auria Lorenza Salcedo Aparicio; Joseline Elizabeth

Pazmiño Peñafiel

Picasso, y también son conocidos numerosos casos de niños prodigio que posteriormente no han desarrollado más habilidades que cualquier otro adulto.

- Genios: hay menos unanimidad respecto a esta denominación, pero bajo este término se encontrarían sujetos con una gran capacidad intelectual y de producción, históricamente el caso más representativo sería Leonardo Da Vinci. También se denominan así a los sujetos de capacidad ilimitada.

- Talentos: El talento es la capacidad de rendimiento superior en un área de la conducta humana.

- Alta capacidad: se reconoce en este grupo a sujetos con alta capacidad intelectual medida a través de pruebas psicométrica.

- Brillantes: sujetos que destacan en alguna capacidad en un contexto determinado. Excepcionales: sujetos que se desvían de la media.

- Superdotado: Algunos autores reservan este término para adultos que destacan en todas las áreas del conocimiento humano.

Uno de los objetivos más importantes de los programas para los superdotados y talentosos es ayudarle a descubrir sus capacidades, que descubran los ámbitos más apropiados para su futuro profesional y científico. Proporcionar a estos estudiantes las diferentes experiencias necesarias para fomentar sus capacidades y utilizar su energía mental en los ámbitos que los ayuden a alcanzar su más alto nivel de auto-afirmación y de excelencia. Otro aspecto clave: el rol tan importante del profesor y su formación en temas relativos a la alta habilidad (Prieto, Abril ). El programa debe orientar a la escuela como institución, a la formación del 


\section{Cuadro comparativo de estudiantes con súper dotación en las carreras de humanidades en las universidades de Perú y Ecuador}

Vol. 3, núm. 3 Esp., (2019)

Denisse Maricela Salcedo Aparicio; Auria Lorenza Salcedo Aparicio; Joseline Elizabeth Pazmiño Peñafiel

profesorado para atender de forma adecuada la diversidad de estos alumnos y a la implementación de programas de enriquecimiento. Es también importante destacar que la implementación exitosa del modelo requiere una alta calidad de los profesores, un alto nivel de compromiso para crecer profesionalmente, disposición para esforzarse a fin de superar los diversos problemas y numerosos desafíos que surgen.

Los test de inteligencia pueden predecir el éxito escolar, pero no el éxito profesional o el éxito en la vida, pues para esto se requieren otras condiciones, como el juicio social, la empatía, la tolerancia a la frustración, la motivación, el autocontrol, la capacidad de esfuerzo y las habilidades de comunicación y de resolución de problemas. Además, existen otras variables que influyen en el rendimiento durante las pruebas de inteligencia, como el interés en la evaluación, la salud física, la autoestima y la resistencia. No está demás decir que los psicólogos tenemos algunos problemas metodológicos, por ejemplo, existen pruebas de inteligencia que no están actualizadas para seguir empleándose en el país. Es muy difícil adquirir pruebas originales y se emplean fotocopias que no siempre están bien reproducidas (Arbaccó, 2019).

Es frecuente observar como los estudiantes con mayores capacidades intelectuales son propensos a sufrir lo que Terrassier llamó el "Efecto Pigmalión negativo". Este se presenta cuando, al haber estudiantes superdotados que presentan un potencial mayor que los profesores, estos últimos tienden a esperar por parte de estos jóvenes un rendimiento en el rango de la media, y pasan entonces a animar a algunos estudiantes a rendir muy por debajo de sus capacidades reales (Alvarez, 2019). 


\section{Cuadro comparativo de estudiantes con súper dotación en las carreras de humanidades en las universidades de Perú y Ecuador}

Vol. 3, núm. 3 Esp., (2019)

Denisse Maricela Salcedo Aparicio; Auria Lorenza Salcedo Aparicio; Joseline Elizabeth

Pazmiño Peñafiel

Características de la personalidad de alumnos superdotados (Ortíz, 2010):

Elevada observación critica

Sienten aburrimiento continuamente.

No les molesta la soledad.

Suelen ser originales, ingeniosos.

Quieren saber el ¿por qué? de las situaciones.

Rinden bien en la escuela si están motivados.

Son muy creativos.
Buen sentido del humor

Marcada incredulidad,

Se hacen preguntas existenciales.

No les gusta someterse a la autoridad.

Generan gran cantidad de ideas, planteamientos.

Dedican más energía para resolver un problema concreto que el promedio.

Fuente: (Ortíz, 2010).

\section{Estudiantes superdotados en Perú}

La nueva Política Nacional de Educación Especial en la Perspectiva de la Educación Inclusiva en Perú (Ministerio de Educación, 2008) subraya la necesidad de proveer condiciones 


\section{Cuadro comparativo de estudiantes con súper dotación en las carreras de humanidades en las universidades de Perú y Ecuador}

Vol. 3, núm. 3 Esp., (2019)

Denisse Maricela Salcedo Aparicio; Auria Lorenza Salcedo Aparicio; Joseline Elizabeth Pazmiño Peñafiel

educativas adecuadas para el desarrollo del alumno superdotado, descrito como aquel que demuestra potencial elevado en cualquier una de las siguientes áreas, aisladas o combinadas: intelectual, académica, liderazgo, psicomotricidad y artes, además de presentar gran creatividad, compromiso para su aprendizaje y la realización de tareas en áreas de su interés (Rodriguez, 2012).

Perú es un país con algunos estudios científicos significativos que apoyan a ciudadanos superdotados. Con la finalidad de propiciar espacios para que faciliten la emergencia de los talentos a lo largo de las diferentes etapas educativas, y tomando en cuenta la necesidad de generar un capital humano de excelencia en aprendizaje e innovación para ser competitivos a nivel internacional, se ha propuesto el proyecto Creatividad, Innovación y Talento (CIT) que reorienta los espacios de intervención, desde el aula de clases en la escolaridad primaria y secundaria, hasta la convocatoria de tipo concurso en la educación superior (Goncalves, 2013).

Este proyecto se plantea en tres niveles de intervención: universitario, secundario y primario. A nivel universitario se plantea realizar un concurso nacional orientado a promover la investigación científica, tecnológica, educativa y humanística en el sector académico, con el fin de propiciar la innovación productiva en el Perú, contribuir a la inserción de nuevas tecnologías a nivel productivo e incentivar la creatividad e innovación en la población nacional, con responsabilidad social. El objetivo principal del proyecto CIT es fomentar la calidad y la excelencia a partir del desarrollo de la competitividad educativa a través de la identificación y apoyo a escolares y universitarios superdotados y talentosos, fomentando el desarrollo de sus 


\section{Cuadro comparativo de estudiantes con súper dotación en las carreras de humanidades en las universidades de Perú y Ecuador}

Vol. 3, núm. 3 Esp., (2019)

Denisse Maricela Salcedo Aparicio; Auria Lorenza Salcedo Aparicio; Joseline Elizabeth

Pazmiño Peñafiel

capacidades de creatividad e innovación con ética y responsabilidad social, aplicado a la ciencia y tecnología, así como al desarrollo educativo, humanístico y social. Para los universitarios talentosos (Blumen, 2008):

- Identificar a los universitarios talentosos peruanos a partir de sus habilidades de generación creativo-productiva, motivación y liderazgo con ética y responsabilidad social, en la educación superior.

- Fomentar y destacar iniciativas innovadoras de los universitarios talentosos, reconociendo casos destacados que deriven en la aplicación productiva.

- Crear pasantías en instituciones y empresas donde los universitarios superdotados y talentosos puedan enriquecerse de experiencias laborales aplicadas en el área de su talento (científico, tecnológico, educativo y humanístico).

- Difundir a nivel nacional e internacional el talento creativo-productivo que existe en el Perú, mediante la participación de los ganadores y finalistas en el libro Talento CreativoProductivo en el Perú 2008, editado por el Fondo Editorial de la Pontificia Universidad Católica del Perú y mediante la participación de los ganadores en el Congreso Mundial de Superdotación y Talento que se realizó en el año 2009.

\section{Estudiantes superdotados en Ecuador}

La población de estudiantes universitarios de Cuenca en Ecuador, da a conocer sobre las estrategias de afrontamiento utilizadas por esta población, donde se constató 4 de 18 estrategias que se utilizan a menudo por los estudiantes, en el área dirigido a la resolución de problemas el 


\section{Cuadro comparativo de estudiantes con súper dotación en las carreras de humanidades en las universidades de Perú y Ecuador}

Vol. 3, núm. 3 Esp., (2019)

Denisse Maricela Salcedo Aparicio; Auria Lorenza Salcedo Aparicio; Joseline Elizabeth Pazmiño Peñafiel

$55 \%$ busca diversiones relajantes, $52 \%$ se fija en lo positivo, el $51 \%$ opto por esforzarse 5 y tener éxito, la distracción física con el $43 \%$. Además un $50 \%$ utiliza un tipo de afrontamiento improductivo como la estrategia de preocuparse (Fajardo, 2019).

Un estudio con 100 estudiantes universitarios, seleccionados al azar en Ecuador, cuya edad de los encuestados fue en un intervalo de 18-29 años de edad, aplicando un test para evaluar su inteligencia. 76 estudiantes universitarios que equivale al $76 \%$, obtiene como resultado el tipo de cociente intelectual que corresponde al término medio, esto evidencia que la población se encuentra en un rango normal, según la calificación obtenida en los resultados de los reactivos aplicados, en este rango se obtuvo una calificación de 20 a 34 aciertos, 18 estudiantes que equivale al $18 \%$ pertenece al tipo de cociente intelectual superior al término medio, estos individuos se encuentran en un rango más arriba del término medio, obteniendo una calificación de 35 a 50 aciertos, 6 participantes equivalente al $6 \%$ el cual se encuentra en un cociente intelectual Inferior al término medio, es decir se encuentra en un rango inferior al término medio, dando como resultado una calificación de 12 a 19 aciertos, no se obtuvo ningún participante que tenga un cociente intelectual superior es decir con una calificación más de 50 aciertos, y en el rango inferior con una puntuación menos de 12 aciertos (Morejón, 2019).

Otro estudio realizado en Ecuador para el año 2019, la cantidad de población estuvo conformada 8 estudiantes de instituciones educativas del Ecuador que poseen las características de superdotados por encontrarse por encima de la media promedio de sus compañeros en rendimiento académico a quienes se les aplicó el test de Herrmann para conocer el cuadrante predominante en su aprendizaje. En cuanto a los resultados se tiene que para el cortical 


\section{Cuadro comparativo de estudiantes con súper dotación en las carreras de humanidades en las universidades de Perú y Ecuador}

Vol. 3, núm. 3 Esp., (2019)

Denisse Maricela Salcedo Aparicio; Auria Lorenza Salcedo Aparicio; Joseline Elizabeth

Pazmiño Peñafiel

izquierdo (CI) se obtuvo un porcentaje del 37\%, para el límbico izquierdo (LI) con un $25 \%$, en cuanto al límbico derecho (LD) un $13 \%$ y para el cortical derecho (CD), un $25 \%$ (Fajardo, 2019).

Los estudiantes con predominancia el cortical izquierdo (CI), de acuerdo Herrmann, son personas con un pensamiento o estilo de aprendizaje concreto, analizan la realidad desde evidencias concretas y suelen tener buen humor a partir de la ironía, podrían destacarse en profesiones relacionadas al cálculo matemático, físico, químico, siendo necesario en la escuela fomentar este tipo de conocimientos con la finalidad de promover las potencialidades de los estudiantes en aras de promover su vocación profesional en pertinencia a su estilo de aprendizaje.

Los participantes con predominancia en el límbico izquierdo (LI), de acuerdo a Ned Herrmann, se caracterizan por ser planificadores y organizadores, por lo general no desarrollan actividades sin que estas se encuentren planificadas y bajo su supervisión. Este es un estilo de aprendizaje que permite fomentar en los estudiantes la vocación profesional hacia carreras que impliquen ser planificadores, estrategas, proyectándose la posibilidad de promover la inteligencia espacial (Fajardo, 2019).

Los estudiantes en relación al límbico derecho (LD), se caracterizan por ser buenos comunicadores y manejadores de las emociones para la movilización de masas, en este sentido, se proyecta un liderazgo carismático transformacional por medio de la motivación, este tipo de estudiantes podrían ser buenos políticos, educadores, líderes religiosos, periodistas, lo cual proyecta que este segmento poblacional se fomenten las competencias 


\section{Cuadro comparativo de estudiantes con súper dotación en las carreras de humanidades en las universidades de Perú y Ecuador}

Vol. 3, núm. 3 Esp., (2019)

Denisse Maricela Salcedo Aparicio; Auria Lorenza Salcedo Aparicio; Joseline Elizabeth Pazmiño Peñafiel

pertinentes para promover la vocación laboral en alguna de estas áreas. Quienes se encuentran en el segmento cortical derecho (CD), se caracterizan por tener un aprendizaje visual, creativo e innovador, se destacan profesiones como la arquitectura, diseño, escritores, filósofos, lo cual genera la posibilidad de fomentar un aprendizaje creativo, dinámico, autónomo en los estudiantes, con la finalidad de promover el auto descubrimiento de sus potencialidades.

\section{Ecuador versus Perú en cifras}

Imagen 1. Distribución comparativa de estudiantes superdotados de Ecuador y Perú

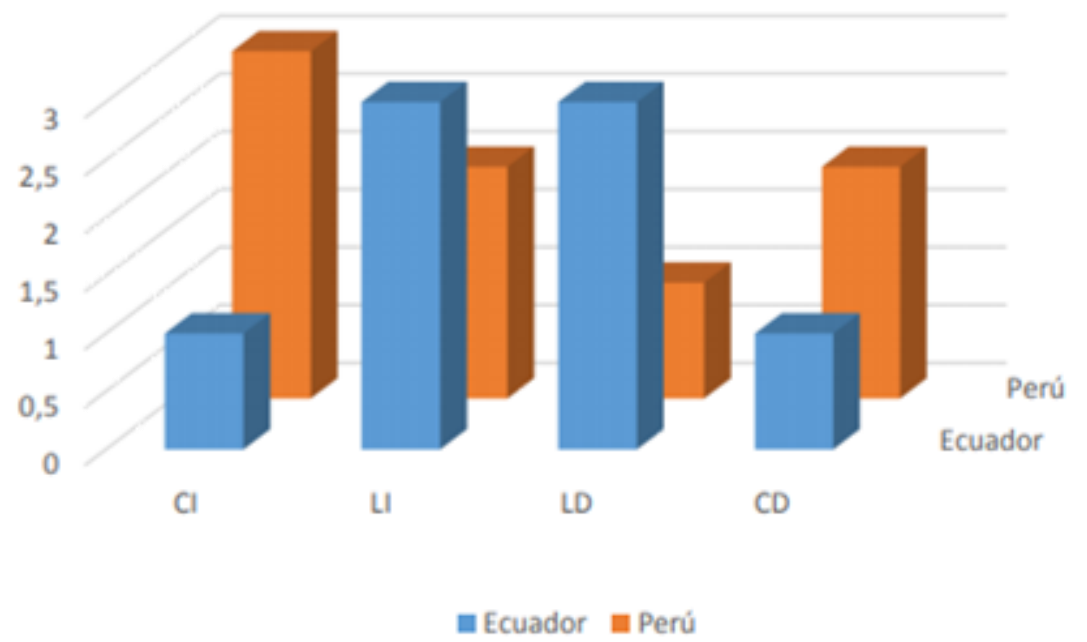

Fuente: (Colcha, 2019).

Los estudiantes de Perú tienen prevalencia en el cortical izquierdo correspondiente con la teoría, son individuos con estilo de pensamiento concreto, con buen humor cargado de ironía, se destacan en profesiones relacionadas con cálculos matemáticos, físicos y químicos, requiriendo que sean aprovechadas al máximo estas áreas en los centros educativos o de formación. Se 


\section{Cuadro comparativo de estudiantes con súper dotación en las carreras de humanidades en las universidades de Perú y Ecuador}

Vol. 3, núm. 3 Esp., (2019)

Denisse Maricela Salcedo Aparicio; Auria Lorenza Salcedo Aparicio; Joseline Elizabeth

Pazmiño Peñafiel

caracteriza por un aprendizaje visual, creativo e innovador. Son carreras idóneas para este perfil la arquitectura, diseño, filosofía, escritura (Colcha, 2019).

Los estudiantes de Ecuador tienen prevalencia al límbico derecho mostrando dominio en áreas afines a la comunicación, inteligencia emocional, carisma y liderazgo para mover masas, este tipo de estudiantes se perfilan para ser políticos, comunicadores sociales, líderes religiosos, educadores. Con prevalencia en el límbico izquierdo, los estudiantes ecuatorianos se caracterizan por ser planificadores, organizadores, por lo general no desarrollan actividades que no estén planificadas y bajo supervisión.

\section{Conclusiones.}

Los test de inteligencia pueden predecir el éxito escolar, pero no el éxito profesional o el éxito en la vida, pues para esto se requieren otras condiciones, como el juicio social, la empatía, la tolerancia a la frustración, la motivación, el autocontrol, la capacidad de esfuerzo y las habilidades de comunicación y de resolución de problemas. Además, existen otras variables que influyen en el rendimiento durante las pruebas de inteligencia, como el interés en la evaluación, la salud física, la autoestima y la resistencia para poder ubicar con algo de certeza a un individuo como superdotado.

Este proyecto, para poder ser exitoso debe plantearse en tres niveles de intervención: universitario, secundario y primario. A nivel universitario se plantea realizar un concurso nacional orientado a promover la investigación científica, tecnológica, educativa y humanística en el sector académico, con el fin de propiciar la innovación. Contribuir a la inserción de nuevas 


\section{Cuadro comparativo de estudiantes con súper dotación en las carreras de humanidades en las universidades de Perú y Ecuador}

Vol. 3, núm. 3 Esp., (2019)

Denisse Maricela Salcedo Aparicio; Auria Lorenza Salcedo Aparicio; Joseline Elizabeth Pazmiño Peñafiel

tecnologías a nivel productivo e incentivar la creatividad e innovación en la población nacional, con responsabilidad social. El objetivo principal del proyecto debe orientarse a fomentar la calidad y la excelencia a partir del desarrollo de la competitividad educativa a través de la identificación y apoyo a escolares y universitarios superdotados y talentosos, fomentando el desarrollo de sus capacidades de creatividad e innovación con ética y responsabilidad social, aplicado a la ciencia y tecnología, así como al desarrollo educativo, humanístico y social.

Otro elemento importante es que mucho de estos jóvenes no logran desarrollar todas sus potencialidades por falta de conocimiento de los docentes y padres, muchos diagnosticados erróneamente como autistas por ser diferentes, lo que lleva en ocasiones a ser personas aisladas en las escuelas por ser diferentes. Se recomienda que los entes educativos tanto del sector privado como público brinden el apoyo y formación a los docentes quienes son los que compartirán gran parte del tiempo con estos jóvenes especiales.

\section{Bibliografía.}

Alvarez, P. (2019). Estudiantes superdotados: diferencias individuales entre niños con una inteligencia extraordinaria. Obtenido de Psicología y Mente: https://psicologiaymente.com

Arbaccó, M. (25 de Octubre de 2019). La inteligencia y los niños superdotados. Obtenido de Diario "El Peruano": https://elperuano.pe

Blumen, S. (2008). Motivación, sobredotación y talento: un desafío para el éxito. Revista de Psicología(26), 147 - 184.

Colcha, R. (2019). Análisis comparativo de los estudiantes superdotados del Ecuador y Perú. Revista Arbitrada Interdisciplinaria KOINONIA Año , 6(8), 710- 21.

Fajardo, Z. (2019). Estilos de aprendizaje en estudiantes superdotados del Ecuador. Revista Interdisciplinaria de Humanidades, Educación, Ciencia y Tecnología, 6(10), 536- 546. 


\section{Cuadro comparativo de estudiantes con súper dotación en las carreras de humanidades en las universidades de Perú y Ecuador}

Vol. 3, núm. 3 Esp., (2019)

Denisse Maricela Salcedo Aparicio; Auria Lorenza Salcedo Aparicio; Joseline Elizabeth

Pazmiño Peñafiel

Goncalves, F. (2013). Creatividad en el aula: percepciones de alumnos superdotados y nosuperdotados. Revista de Psicología , 31(1), 37-66.

Martínez, E. (02 de Octubre de 2019). Niños superdotados. Obtenido de Mapfre: https://www.salud.mapfre.es

Morejón, L. (Febrero de 2019). Inteligencia y estrategias de afrontamiento al estrés. Obtenido de Reposorio Digital: http://repositorio.uta.edu.ec

Ortíz, E. (2010). Los estilos de aprendizaje, la superdotación intelectual y el talento en estudiantes universitarios. Revista Estilos de Aprendizaje, 5(3), 84-100.

Prieto, M. (2010 de Abril ). Alta habilidad: superdotación y talento. Revista Electrónica Interuniversitaria de Formación del Profesorado, 13(1), 15-20.

Rodriguez, R. (2012). Aceleración de la enseñanza para alumnos superdotados: argumentos favorables y contrarios. Revista de Psicología , 30(1), 189-214.

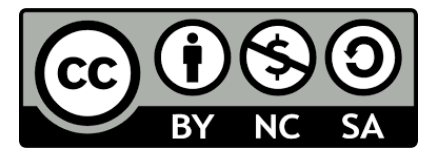

RECONOCIMIENTO-NOCOMERCIAL-COMPARTIRIGUAL

CC BY-NC-SA

ESTA LICENCIA PERMITE A OTROS ENTREMEZCLAR, AJUSTAR Y CONSTRUIR A PARTIR DE SU OBRA CON FINES NO COMERCIALES, SIEMPRE Y CUANDO LE RECONOZCAN LA AUTORÍA Y SUS NUEVAS CREACIONES ESTÉN BAJO UNA LICENCIA CON LOS MISMOS TÉRMINOS. 